\title{
In Situ Tissue Imaging and Monitoring Technique using WGM Probes
}

\section{Punthawanunt $\mathbf{S}^{1}$ and Yupapin $\mathrm{PP}^{1,2 \star}$}

${ }^{1}$ Interdisciplinary Research Center, Faculty of Science and Technology, KasemBundit University, Thailand

${ }^{2}$ Nano Research Center, Faculty of Engineering, Ton DucThang University, Ho Chi Minh City, Vietnam

\section{Opinion}

Micro and Nano devices have been used as the interesting tools for many research and applications [1-3], where there are many available techniques today. From which Yupapin and his colleagues used the technique known as whispering gallery mode (WGM) of light within a tiny system that has shown the promising advanced in many applications in either theoretical and practical applications [47], eMicro-conjugate mirror has been the interesting device for $3 \mathrm{D}$ imaging construction, where it was given in reference. The 3D image can be constructed and obtained on a tip of the light cone (WGM) outputs as shown in Figure 1, where the interesting aspect is that the reflect output of the opposite object is formed by the conjugate mirror construction, where the $3 \mathrm{D}$ output is obtained and confirmed by the four wave mixing concept, which is the nonlinear effect obtained by using the micro-conjugate mirror. The dual property probes are based on $3 \mathrm{D}$ aiming structure, where the particle/light probes can be used, which are (1) tunneling probe and (2) WGM probe, from which the in situ monitoring can be applied. The tunneling particle collection is centered by the WGMs, where the WGM probes are penetrated into the different depths, where the depth resolution can be classified by the probe sizes in either WGM or electron. One of them has shown the use of WGM light beam to form a probe for 3D imaging applications, in which the important function of this device is the real time 3D imaging, which can be used to form the required in situ operation. Recently, the use of such a device for in situ imaging has shown the very interesting work [5]. In this commentary, the use of WGM probes for multi layers imaging is designed and described for in situ imaging usage, which is targeted to be the tiny device that can be used as the portable equipment. In Figure 1, the WGM probe is generated and formed by using the nonlinear micro ring resonator, which is the known as a Panda ring resonator. Light from a commercial semiconductor laser (or an embedded laser) is input into the input port, where the WGM probe can be generated by the suitable controlled parameters.

In application, the two-side WGM probes can be formed, in which one side is used for imaging probing, while the other one for the $3 \mathrm{D}$ image output, which is the real time imaging that can be seen directly (or on display screen), thus, the in situ imaging can be applied. In case of large scale sample, the large scale scanning can also be applied, where the device will be formed by the array or distributed forms. From which the distributed sensing system can be used to detect the large area sample, where the real images can be seen on the other side pixels. The 3D images can be seen by means of in situ monitoring, which can be used for tissue structure monitoring, blood flow measurements, where the bi-directional WGM probe is the key concept. From Figure 2, the sample depths can be measured by using the multi-sensing probes (heads), which can be integrated and used as the portable equipment. In operation, the projection of different sample depths with different incident and reflected light powers on the sample layer is performed. The reflected powers are reversed back into the different ring resonators within the same devices, from which the different sample depths can be distinguished (measured) and displayed by the drop ports and in situ imaging panel (Figure 1).
The imaging construction can also be done by the drop port output signals, from which the 3D imaging can be constructed and seen. However, the real time 3D images can be seen on the back panel, which is constructed by each pixel tips of the WGM probes, where the large area (volume) of 3D pixel imaging spots can be used to construct the whole image [2]. Moreover, such a device can be used for medical diagnosis and therapy. For further applications, such a concept of

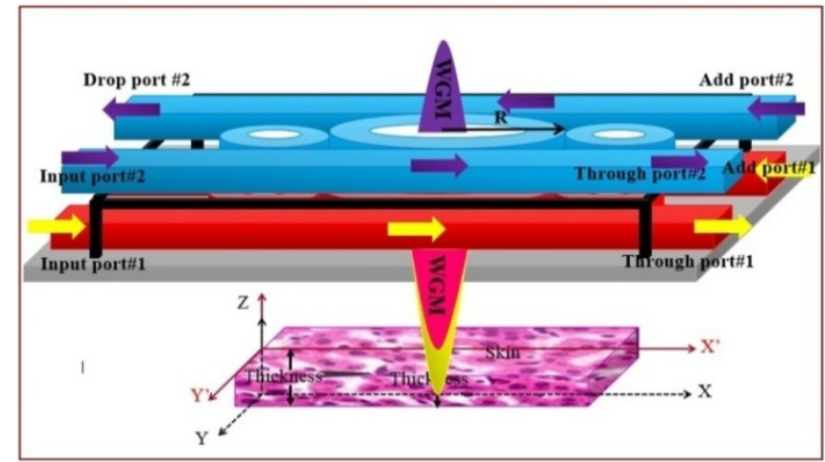

(a)

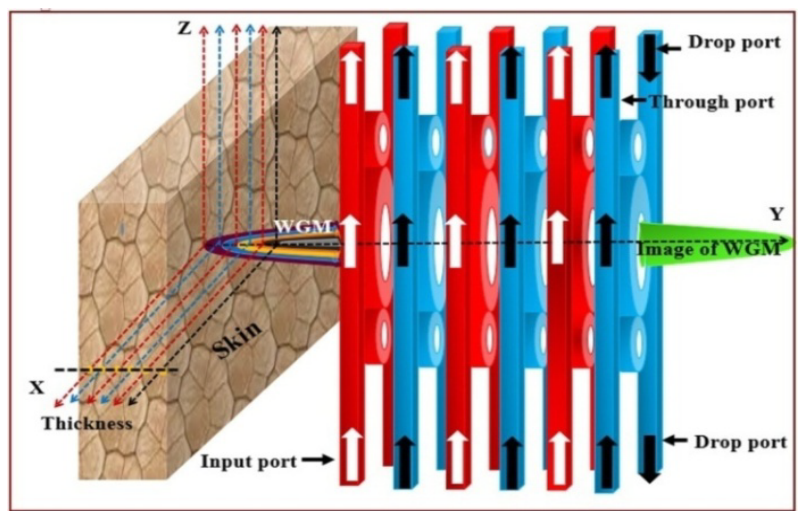

(b)

Figure 1: Multi-WGM probes scanning for tissue imaging and monitoring, where (a) WGM probe generated by a Panda ring device, (b) Multi-layers imaging system.

${ }^{*}$ Corresponding author: Yupapin PP, Interdisciplinary Research Center, Faculty of Science and Technology, Kasem Bundit University, Bangkok 10250, Thailand, Tel: +6623202777; E-mail: preecha.yu@kmitl.ac.th

Received June 09, 2016; Accepted June 10, 2016; Published June 20, 2016

Citation: Punthawanunt S, Yupapin PP (2016) In Situ Tissue Imaging and Monitoring Technique using WGM Probes. J Biosens Bioelectron 7: 211. doi:10.4172/2155-6210.1000211

Copyright: () 2016 Punthawanunt S, et al. This is an open-access article distributed under the terms of the Creative Commons Attribution License, which permits unrestricted use, distribution, and reproduction in any medium, provided the original author and source are credited. 
Citation: Punthawanunt S, Yupapin PP (2016) In Situ Tissue Imaging and Monitoring Technique using WGM Probes. J Biosens Bioelectron 7: 211.

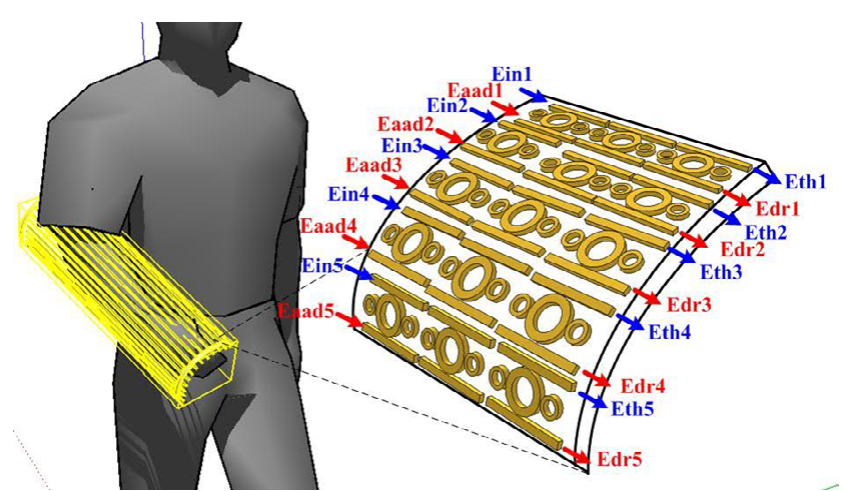

Figure 2: Distributed in situ tissue imaging panel using the Panda ring array (thin film).

WGM probe generated by the distributed sensors can also be used for various applications, for instance, heat thin film source for clothing application, 3D imaging and multi-purpose storage devices, where the hot and cold thin film panel can be constructed on the fabric and formed the thermal control cloth usages (Figure 2).

In conclusion, it is shown that the use of a micro-conjugate mirror for 3D imaging constructing device is possible and available for various applications. The use of a tiny device or panel for 3D imaging as the portable device application is very the interesting equipment, especially, for the in situ 3D imaging requirement, for instance, the tissue structure imaging for diagnosis or operation, where the portable equipment has shown the advantage. Therefore, the 3D imaging instrument is now down the size and brought easily to the patient place. The other applications are also interesting by the same principle, where the in situ imaging is required.

The author would like to acknowledge the KasemBundit University, Bangkok, Thailand and Ton DucThang University, Vietnam for the research facilities.

\section{References}

1. Tamee K, Chaiwong K, Yothapakdee K, Yupapin PP (2015) 3D Imaging Generated by Micro-Optical Devices System with Distortion Improvement. J ICIC Express Letters 8: 707-711.

2. Punthawanunt S, Yupapin PP, Patharacorn $P$ (2015) Novel Design of 3D Imaging Pixel On-chip using Micro-conjugate Mirror System. Nano Biomedicine and Engineering 7: 52-61.

3. Suwandee S, Yupapin PP (2015) Drug Targeting Model of Composite Gold-Tourmaline for Cells Enhancing Applications. Nano Biomedicine and Engineering 7: 38-46.

4. Sarapat N, Frank TD, Yupapin PP (2013) Conjugate Mirror Design and Simulation using a Nonlinear Coupling Microring Circuit. Journal of Nonlinear Optical Physics and Materials 22: 1-11.

5. Sappajak S, Patharacorn P, Chaingga S, Yupapin PP (2016) Multi WGM Light Probes Generated by Distributed Panda Resonator System. Far East Journal of Electronics and Communication 16: 427-442.

6. Yupapin PP (2013) Tissue Culture with 3D Monitoring by Distributed Ring Circuits. Journal of Biosensors and Bioelectronics 4: e119.

7. Thammawongsa N, Yupapin PP (2016) Remote Artificial Eyes using microoptical Circuit for Long-Distance 3D imaing Perception. Artificial Cells Nanomedicine and Biotechnology 44: 106-110. 